nous avons trouvé, pour la constante de vitesses caractérisant le pouvoir catalytique de l'acide sulfophénylacétique, les valeurs:

$\begin{array}{lrrr}\text { pour } t & 1290 & 2730 & 7240 \\ K .10^{4} & 1,24 & 1,05 & 1,00 \text { moyenne } 1,00\end{array}$

Comme elles sont bien inférieures à celles de $K .10^{4}$ pour l'acide chlorhydrique et l'acide sulfurique qui pour $t=2850$ ont été respectivement 3,3 et 2,3, il faut conclure que la présence dans le catalyseur d'un groupement commun au corps à transformer ne confère au catalyseur aucune activité chimique supplémentaire. Bien plus, l'acide benzènesulfonique accélérant la saponification autant que les acides minéraux, l'incorporation à ce composé d'un groupe acide gras a même contribué à diminuer fortement son efficacité. Dans les sulfo-acides utilisés pour la saponification des huiles et des graisses, cette diminution se trouve donc compensée et au-delà par l'action très favorable exercée par ce même groupement sur un facteur purement physique, la solubilité mutuelle des deux phases du système.

Laboratoire de Chimie technique et théorique de l'Université de Genève.

\title{
Schwefel als Brückenatom im Mittelring einiger Anthracenabkömmlinge I
}

\author{
von \\ A. Bistrzyeki und B. Brenken.
}

(27. XI. 21.)

Leitet man in die schwach erwärmte Lösung äquimolekularer Mengen von Thiobenzilsäure ${ }^{1}$ ) und Benzaldehyd trockenes Salzsäuregas ein, so scheidet sich alsbald der Benzylidenätherester der Thiobenzilsäure aus, wie wir vor kurzem gezeigt haben ${ }^{2}$ ). Wir haben diesen cyclischen Ätherester,

$$
\begin{array}{r}
\left(\mathrm{C}_{6} \mathrm{H}_{5}\right)_{2} \mathrm{C} \cdot \mathrm{O} \\
\mathrm{COO}
\end{array}>\mathrm{CH} \cdot \mathrm{C}_{6} \mathrm{H}_{5}
$$

1) Becker und Bistrzycki, B. 47, 3149 (1914); Bistrzycki und Brenken, Helv. 3, 467 (1920).

2) Helv. 3, 455 (1920). 
der sich durch überraschende Beständigkeit gegen manche Reagenzien auszeichnet, 2,4,4-Triphenyl-1,3-oxthiophanon-5 genannt und sein Verhalten ziemlich eingehend beschrieben. Dabei erwähnten wir bereits, dass er unter dem Einflusse von konz. Schwefelsäure eine rätselhafte Umwandlung erleidet, die aufzuklären uns inzwischen gelungen ist, und über die im folgenden berichtet werden soll.

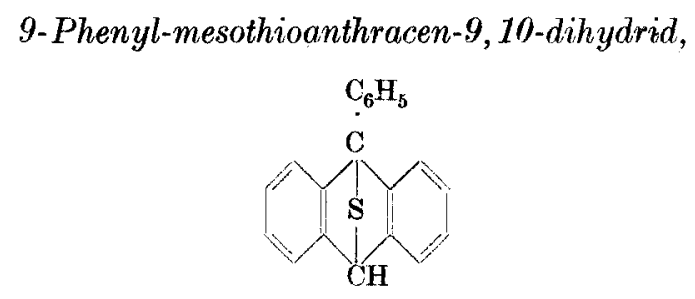

Übergiesst man $1 \mathrm{gr}$ staubfein zerriebenes Triphenyl-oxthiophanon mit 30 bis $40 \mathrm{~cm}^{3} \mathrm{konz}$. Schwefelsäure (spez. Gew. $1,84=94 \%$ ), so löst es sich allmählich unter starker Gasentwicklung (s. u.) mit anfangs gelber Farbe, die schnell in ein tiefes, violettstichiges Rot übergeht. Nach ein- bis zwei-stündigem Stehen, bisweilen auch schon nach einigen Minuten tritt ein schwacher Geruch nach Schwefeldioxyd auf. Wird die Lösung nach eintägigem Stehen durch Glaswatte in $100 \mathrm{~cm}^{3}$ kaltes Wasser filtriert, so scheidet sich ein kanariengelber Körper aus, der grösstenteils in der Flüssigkeit suspendiert bleibt und sich weder nach monatelangem Stehen, noch beim Rühren mit der Turbine absetzt. Seine Isolierung gelang schliesslich, indem man die schwefelsaure Lösung mit konz. Ammoniakwasser neutralisierte und hierauf so lange kochte, bis die Ausflockung begann. Beim Erkalten setzt sich dann ein Niederschlag ab, der abgesaugt, jedoch nicht ausgewaschen (er verschmiert dabei sofort), sondern ohne weiteres auf Ton getrocknet wird. Zur weiteren Reinigung ist es unerlässlich, dieses Rohprodukt mit etwa $5 \mathrm{~cm}^{3}$ kaltem Methylalkohol zu digerieren; man giesst dann die braune, grün fluorescierende Lösung ab und krystallisiert den Rückstand aus Aceton + Wasser um. So erhält man gelbe, glänzende, feine Nädelchen, die bei $117^{0}-118^{\circ}$ schmelzen, in einer Ausbeute von höchstens 0,34 gr.

Zur Darstellung grösserer Mengen des gelben Produktes ist es nicht angängig, mehr als 1 gr auf einmal in der konz. Schwefelsäure zu lösen, da auf diese Weise keine wesentlich grössere Menge erhalten werden würde. Man muss vielmehr in verschiedenen Gefässen je $1 \mathrm{gr}$ der Substanz mit der Schwefelsäure übergiessen und vereinigt erst nach 24 Stunden je zwei bis drei der erhaltenen Lösungen zur Weiterver- 
arbeitung. Die Gewinnung der für die vorliegende Arbeit notwendigen Substanzmenge gestaltete sich daher bei Verwendung von etwa $80 \mathrm{mal}$ 1 gr Ausgangsmaterial ziemlich zeitraubend.

Die Verbindung löst sich bei Siedehitze leicht in Benzol oder Chloroform, mässig in Aceton, ziemlich schwer in Eisessig, schwer in Methylalkohol oder Ligroïn, noch schwerer in Äthylalkohol. Alle diese Lösungen fluorescieren grün, am schönsten die in Eisessig. Von kalter konz. Schwefelsäure wird die Substanz mit orangebrauner Farbe aufgenommen Giesst man diese Lösung nach einigen Minuten in kaltes Wasser, so fällt das Produkt unverändert wieder aus. In Alkalilaugen ist es unlöslich.

$0,1586 \mathrm{gr}$ Subst. gaben $0,4872 \mathrm{gr} \mathrm{CO}_{2}$ und $0,0745 \mathrm{gr}_{2} \mathrm{O}$

$0,1376 \mathrm{gr}$ Subst. gaben $0,1145 \mathrm{gr} \mathrm{BaSO}_{4}$.

$$
\begin{aligned}
& \mathrm{C}_{20} \mathrm{H}_{14} \mathrm{~S} \quad \text { Ber. C 83,86 H 4,93 } \mathrm{S} \quad 11,21 \% \\
& \text { Gef. , } 83,78 \quad: 5,25 \quad, 11,43 \%
\end{aligned}
$$

0,6776 gr Subst. gaben (nach Landsberger), in 23,4796 gr Benzol gelöst, eine Siedepunktserhöhung von $0,272^{\circ}$.

$$
\mathrm{C}_{20} \mathrm{H}_{14} \mathrm{~S} \quad \text { Ber. } \mathrm{M}=\mathbf{2 8 6} \text { Gef. } \mathrm{M}=290 .
$$

Das Produkt ist also aus dem Ausgangsmaterial durch Abspaltung der Atome $\mathrm{CH}_{2} \mathrm{O}_{2}$ entstanden. Um zu entscheiden, ob diese Zusammensetzungsdifferenz als $\mathrm{CO}_{2}+\mathrm{H}_{2}$ oder als $\mathrm{CO}+\mathrm{H}_{2} \mathrm{O}$ aufzufassen wäre, wurde die Reaktion zwischen Triphenyl-oxthiophanon und konz. Schwefelsäure in einem geschlossenen Kölbchen vorgenommen unter Durchleiten eines kohlendioxydfreien, trockenen Luftstromes, der sodann eine Jod-Kaliumjodidlösung, ein Calciumchloridrohr und schliesslich einen gewogenen Kaliapparat passierte. Es zeigte sich, dass kein Kohlendioxyd abgespalten wurde. Als dagegen dieser Versuch im Kohlendioxydstrom vorgenommen und das entweichende Gas über Kalilauge aufgefangen wurde $^{1}$ ), ergab sich, dass etwa ein Drittel der Menge Kohlenoxyd abgespalten wurde, die der Bruttogleichung $\mathrm{C}_{21} \mathrm{H}_{16} \mathrm{O}_{2} \mathrm{~S}-\mathrm{CO}-\mathrm{H}_{2} \mathrm{O}=$ $\mathrm{C}_{20} \mathrm{H}_{14} \mathrm{~S}$ entspricht, also in guter Übereinstimmung mit der Ausbeute an dem reinen gelben Produkt ${ }^{2}$.

$0,2048 \mathrm{gr}$ Subst. gaben $4,8 \mathrm{~cm}^{3}$ feuchtes Kohlenoxyd $\left(12^{\circ}, 716 \mathrm{~mm}\right)$

$$
\mathrm{C}_{21} \mathrm{H}_{16} \mathrm{O}_{2} \mathrm{~S}-\mathrm{CO} \text { Ber. CO } 8,42 \% \text { Gef. CO } 2,60 \% \text {. }
$$

1) Die Anordnung des Versuches war genan die gleiche wie bei den Entcarbonylierungsversuchen von Bistrzycki und $v$. Siemiradzki, B. 39, 53 (1906), oder Bistrzycki und Ryncki, Mém. Soc. fribourgeoise Sciences nat. Série Chimie 3, 169 (1913). Vergl. auch H. Meyer, Analyse und Konstitutionsermittlung organischer Verbindungen, IIT. Aufl. S. 598 (1916).

2) Verwendet man an Stelle von 94-proz. Schwefelsäure die absolute Säure, so erhält man noch ein wenig mehr Kohlenoxyd:

$0,2180 \mathrm{gr}$ Subst. gaben, bei $725 \mathrm{~mm}$ Barometerstand und $21^{\circ}, 6,8 \mathrm{~cm}^{3}$ feuchtes Kohlenoxyd.

$$
\mathrm{C}_{21} \mathrm{H}_{16} \mathrm{O}_{2} \mathrm{~S}-\mathrm{CO} \text { Ber. CO } 8,42 \% \text { Gef. CO } 3,38 \% \text {. }
$$


Hiervon ausgehend, glauben wir die Entstehung dieser gelben Verbindung folgendermassen deuten zu können:

Der Benzylidenätherester der Thiobenzilsäure nimmt unter Sprengung des Fünf-Ringes eine Molekel Wasser (oder vielleicht Schwefelsäure) auf, indem sich die entsprechende Thioäthersäure bildet. Diese spaltet unter dem Einfluss der konz. Schwefelsäure, ähnlich der Thiobenzilsäure selbst'1), Kohlenoxyd ab:

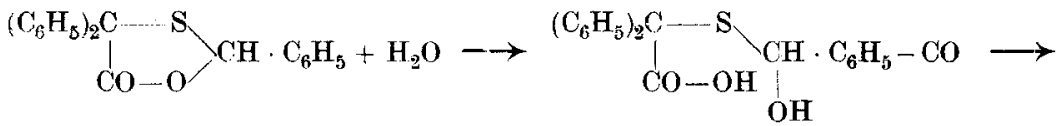

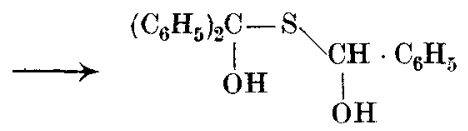

Der hierbei gebildete Thioäther erleidet weiter unter Abgabe von zwei Molekeln Wasser eine innere Kondensation, die zu einem geschwefelten Derivate des Phenylanthracen-dihydrids führt:

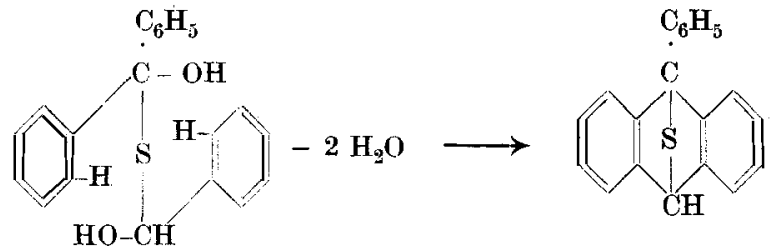

Mit dieser Anschauung steht das chemische Verhalten des Produktes in vollem Einklang.

Destilliert man $5 \mathrm{gr}$ des gelben Körpers in Anteilen von 0,5 gr in üblicher Weise ${ }^{2}$ ) über glühenden Zinkstaub, so erhält man rotgelbe Destillate, die in abs. Alkohol gelöst wurden. Die braun gefärbten Lösungen wurden vereinigt, eine halbe Stunde mit'Tierkohle gekocht und filtriert. Beim Erkalten schieden sich glänzende, gelblich gefärbte Blättchen aus, deren Menge nach zweimaligem Umkrystallisieren aus abs. Alkohol 1,2 gr betrug. Sie erwiesen sich als völlig schwefelfrei und als identisch mit dem 9 -Phenylanthracen ${ }^{3}$ ). Wie dieses, schmolzen sie bei $152^{\circ}$, fluorescierten in stark verdünnter benzolischer oder alkoholischer Lösung intensiv blauviolett und gaben, in Benzollösung mit

1) Becker und Bistrzycki, B. 47, 3151 (1914).

2) Vergl. z. B. Levy und Bistrzycki, Anleitung zur Darstellung organisch-chemischer Präparate, IV. Aufl., S. 208. Stuttgart, Enke. 1902.

3) Baeyer und Schillinger, A. 202, 61 (1880). 
Pikrinsäure zusammengebracht, eine dunkelrote Lösung, aus der sich beim Verdunsten über Paraffin ein rotes Pikrat abschied.

$$
\begin{aligned}
& 0,1527 \text { gr Subst. gaben } 0,5274 \mathrm{gr} \mathrm{CO}_{\mathrm{a}} \text { und } 0,0772 \mathrm{gr}^{\mathrm{H}} \mathrm{H}_{2} \mathrm{O} \\
& \mathrm{C}_{20} \mathrm{H}_{14} \quad \text { Ber. C 94,45 H 5,55\% } \\
& \text { Gef. ,, 94,20 , ,5,68\% }
\end{aligned}
$$

Bei der Zinkstaubdestillation war also der Schwefel aus der Molekel herausgenommen worden.

Dies gab Anlass zu erwägen, ob der Schwefel überhaupt in der angenommenen Bindung vorhanden sei, oder ob das gelbe Produkt vielleicht der Formel I entspräche, die ja durch Atomverschiebung, viel-

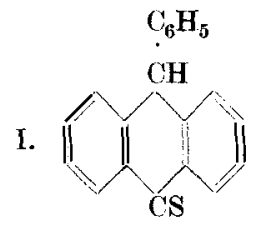

II.

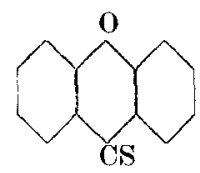

lescht auch direkt hätte zustandegekommen sein können. In diesem Falle würde die Verbindung ein cyclisches Thioketon vorstellen, wie etwa das Xanthion (II). Ihre Beständigkeit spricht jedoch gegen diese Auffassung. Während das Xanthion nämlich unter der Einwirkung von Phenylhydrazin ${ }^{1}$ ) oder von alkoholischer Natronlauge ${ }^{2}$ ) seinen Schwefel leicht abspaltet, blieb unsere Substanz unverändert, als sie fünf Stunden in alkoholischer Lösung mit Phenylhydrazin oder eine Stunde mit etwa normaler wässrig-alkoholischer Kalilauge gekocht wurde. Auch cinstündiges Kochen mit Anilin liess sie völlig unverändert.

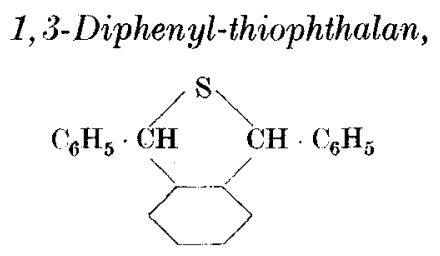

$1 \mathrm{gr}$ Phenyl-mesothioanthracen-dihydrid wird, in $20 \mathrm{~cm}^{3}$ Eisessig gelöst, mit $10 \mathrm{gr}$ geraspeltem Zink versetzt und unter allmählichem Zusatz von $5 \mathrm{~cm}^{3}$ rauchender Salzsäure bis zur fast vollständigen Entfärbung der Lösung (etwa 1 bis 11/2 Stunden lang) unter Rückfluss gekocht. Aus der filtrierten lösung fällt auf Wasserzusatz ein weisser,

1) Graebe und Röder, B. 32, 1690 (1899).

2) R. Meyer und Szanecki, B. 33, 2580 (1900). 
krystallinischer Niederschlag, der sich, gleich der Lösung selbst, beim Stehen an der Luft grünlich-gelb färbt. Wird er aber ohne Verzug dreimal aus abs. Alkohol umkrystallisiert, so gelangt man zu farblosen, seidenglänzenden Nadeln (0,3 gr) vom Smp. 106,5-107,50, deren Molekel der Analyse nach zwei Wasserstoffatome mehr als die des Ausgangsmaterials enthält.

0,1483 gr Subst. gaben $0,4512 \mathrm{gr} \mathrm{CO}_{2}$ und $0,0788 \mathrm{gr}_{2} \mathrm{O}$

$0,1687 \mathrm{gr}$ Subst. gaben $0,1390 \mathrm{gr} \mathrm{BaSO}_{4}$

$$
\begin{array}{llll}
\mathrm{C}_{20} \mathrm{H}_{16} \mathrm{~S} & \text { Ber. C 83,28 } & \text { H } 5,59 & \text { S } 11,13 \% \\
& \text { Gef. , } 82,98 & \text {, } 5,94 & \text {, 11,37\% }
\end{array}
$$

Wäre das gelbe Produkt ein Thioketon, so wäre entweder die völlige Eliminierung des Schwefels oder die Bildung eines Mercaptans zu erwarten gewesen. Mercaptancharakter besitzt die Verbindung jedoch nicht; sie wird durch eine alkoholische Jodlösung ${ }^{1}$ ) nicht oxydiert und gibt auch mit Ferrichlorid keine Reaktion. Wir glauben, dass sie aus dem Phenyl-mesothioanthracen-dihydrid durch Aufspaltung des Mittelringes entstanden ist:

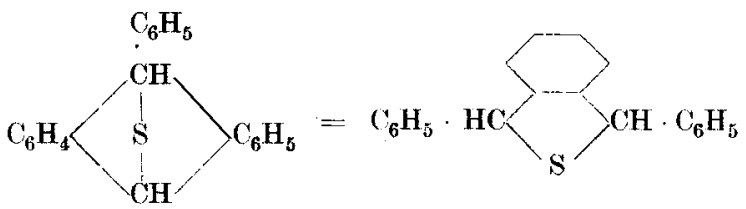

Demnach ist die Verbindung als ein diphenyliertes o-Xylylensulfid oder Diphenyl-thiophthalan zu betrachten ${ }^{2}$ ). Diese Auffassung beruht auf dem Ergebnis der weiteren Reduktion des Phenyl-mesothioanthracendihydrids.

Kocht man nämlich eine Lösung von $1 \mathrm{gr}$ desselben in $20 \mathrm{~cm}^{3}$ Eisessig mit 10 gr Zinkstaub und zwar der Sorte ,Extrafein"3) von Kahlbaum unter allmählichem Zusatz von 6 bis $8 \mathrm{~cm}^{3}$ rauchender Salzsäure am Rückflusskühler $3 \frac{1}{2}$ bis 4 Stunden lang, so entweicht Schwefelwasserstoff in reichlicher Menge. Aus der jetzt völlig entfärbten, filtrierten Lösung fällt auf Wasserzusatz ein farbloses Produkt aus, das nach zweimaliger Krystallisation aus Alkohol + Wasser feine, weisse Prismen $(0,25 \mathrm{gr})$ bildet und aus dem gelben.Ausgangsprodukt unter

1) Vergl. Klason und Carlson, B. 39, 739 (1906).

2) Zur Nomenklatur vergl. Meyer-Jacobson, Lehrbuch II, 3, S. 143; zur Bezifferung ebenda, S. 35 .

3) Diese Sorte hat sich auch bei anderer Gelegenheit viel wirksamer gezeigt als der gewöhnliche Zinkstaub. 
Abspaltung des Schwefels und Aufnahme von vier Wasserstoffatomen entstanden ist. (Die Isolierung dieses Produktes glückt allerdings nicht immer.)

0,1550 gr Subst. gaben $0,5268 \mathrm{gr} \mathrm{CO}_{2}$ und $0,0984 \mathrm{gr}_{2} \mathrm{O}$

$$
\begin{array}{lll}
\mathrm{C}_{20} \mathrm{H}_{1 \mathrm{~b}} & \text { Ber. C 92,98 } & \text { H 7,02\% } \\
& \text { Gef. .: 92,70 } & \text { "7,10\% }
\end{array}
$$

Diese Verbindung konnte identifiziert werden mit dem bereits von Zincke $e^{1}$, Radisiewanowsk $i^{2}$ ) und Kohler ${ }^{3}$ ) dargestellten o-Dibenzylbenzol, für das die Autoren den Smp. $78^{\circ}$ angeben, welchen auch unser Produkt aufweist.

Dieselbe Verbindung entsteht auch bei weiterer Reduktion des Diphenyl-thiophthalans mit ,extrafeinem“ Zinkstaub (wie oben), was die für dieses angenommene Konstitution beweist.

Wäre die Reduktion des Phenyl-mesothioanthracen-dihydrids unter Aufspaltung nicht an dem Kohlenstoffatom 9, sondern an 10 erfolgt, so hätte sie zum Diphenyl-o-tolylmethan führen müssen, das bekannt ${ }^{4}$ ) (Smp. $83^{\circ}$ ) und mit dem Reaktionsprodukt nicht identisch ist.

In Übereinstimmung mit unserer Auffassung des Reduktionsverlaufes steht auch das Ergebnis der Oxydation: Eine warme Lösung von $0,8 \mathrm{gr}$ Phenyl-mesothioanthracen-dihydrid in $15 \mathrm{~cm}^{3}$ Eisessig wird mit 1,5 gr 30-proz. Wasserstoffsuperoxyd (Perhydrol) versetzt und bis zum Eintritt einer Reaktion erwärmt, worauf man die Flamme entfernt. Die anfangs gelbe, grün fluorescierende Lösung färbt sich dabei unter starker Gasentwicklung zunächst dunkelbraun, wird aber bei fortschreitender Reaktion wieder heller. Sollte nach Zugabe eines weiteren Grammes Perhydrol beim Erwärmen noch Reaktion eintreten, so wäre abermals ein wenig $(0,5 \mathrm{gr})$ Perhydrol zuzufügen und aufzukochen. Schliesslich wird das Reaktionsgemisch 5 Minuten lang unter Rückfluss zum Sieden erhitzt, abgekühlt und mit Wasser versetzt. Der hierbei ausfallende krümelige Niederschlag $(0,6 \mathrm{gr})$ ist orangebraun, kann aber durch wiederholtes Kochen seiner alkoholischen Lösung mit Tierkohle entfärbt werden, wenn auch nur unter erheblichen Verlusten. Man erhält so farblose, mikroskopische, vierseitige Prismen vom Smp. 146-148º.

\footnotetext{
1) B. 6, 119ff. (1873); 9, 31 (1876).

2) B. 27, 3237 (1894).

3) Am. 40, 227 (1908).

4) Bistryycki und Gyr, B. 37, 1249 (1904).
} 
Sie sind identisch mit dem o-Dibenzoylbenzol $\mathrm{C}_{6} \mathrm{H}_{4}\left(\mathrm{CO} \cdot \mathrm{C}_{6} \mathrm{H}_{5}\right)_{2}$, das zuerst $Z$ incke ${ }^{1}$ ) mit dem Smp. 145-1460 durch Oxydation von o-Dibenzylbenzol erhalten hat.

0,1589 gr Subst. gaben $0,4873 \mathrm{gr} \mathrm{CO}_{2}$ und $0,0715 \mathrm{gr}_{2} \mathrm{O}$

$$
\begin{array}{lll}
\mathrm{C}_{2 v} \mathrm{H}_{14} \mathrm{O}_{2} & \text { Ber. C 83,89 } & \text { H 4,93\% } \\
& \text { Gef. ., 83,64 } & \text {, 5,03\% }
\end{array}
$$

Neuerdings ist das gleiche Produkt mit dem Smp. $148^{\circ}$ von Simonis und Remmert ${ }^{2}$ ) durch Oxydation von 9,10-Diphenylanthracen erhalten worden, indem der Mesoring an den Kohlenstoffatomen 9 und 10 gesprengt wurde, ähnlich wie wir es oben für die Reduktion des Phenylmesothioanthracen-dihydrids angenommen haben, und wie es offenbar für die Oxydation des letzteren zutrifft. Durch die Bindung der Kohlenstoffatome 9 und 10 an eine, beziehungsweise zwei Phenylgruppen ist demnach die Festigkeit des Mesoringes gelockert worden.

Wir konnten die Identität unseres Präparates mit dem o-Dibenzoylbenzol von Simonis und Remmert durch einen direkten Vergleich sowie durch den Smp. der Mischung sicherstellen, nachdem Herr Prof. Dr. Simonis die Güte gehabt hatte, uns eine Probe seines Präparates zu übersenden.

Fast alle der früher beschriebenen cyclischen Ätherester der Thiobenzilsäure geben unter der Einwirkung von konz. Schwefelsäure gelbe Produkte, die dem Benzaldehydderivat analog konstituiert sein dürften. Rein dargestellt wurde bisher nur noch das

9-Phenyl-mesothio-2-chlor-anthracen-9, 10-dihydrid,

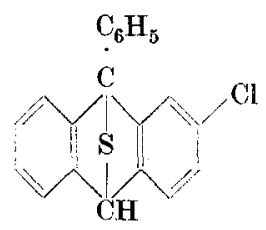

aus dem 2-(p-Chlorphenyl)-4,4-diphenyl-1,3-oxthiophanon-5 (dem Ätherester aus pChlorbenzaldehyd) ${ }^{3}$ ) und zwar in ganz analoger Weise, wie das eingangs beschriebene (chlorfreie) Benzaldehydderivat. Das gelbe Rohprodukt $(0,4 \mathrm{gr})$ wurde zur Reinigung einmal aus Aceton + Wasser, sodann noch dreimal in der Weise krystallisiert, dass

1) B. 9, 32 (1876).

2) B. 48, 208 (1915).

3) Bistrzycki und Brenken, Helv. 3, 458 (1920). 
man die Verbindung in Aceton löste, Alkohol bis zur beginnenden Ausfällung und dann noch etwas Wasser hinzusetzte. Mikroskopische, zu dichten, konzentrischen Büscheln vereinigte, gelbe Prismen; sie erweichen von $120^{\circ}$ an und schmelzen von $124-126^{\circ}$. In der Siedehitze leicht löslich in Benzol, Chloroform oder Ligroïn, ziemlich leicht in Aceton oder Eisessig, sehr schwer in Alkohol. Alle diese Lösungen fluorescieren grün. Konz. Schwefelsäure löst die Verbindung mit brauner, schwach grïnschimmernder Farbe auf, die beim Erwärmen in ein tiefes Violettbraun übergeht.

$$
\begin{aligned}
& 0,1497 \mathrm{gr} \text { Subst. gaben } 0,4103 \mathrm{gr} \mathrm{CO}_{2} \text { und } 0,0601 \mathrm{gr} \mathrm{H}_{2} \mathrm{O} \\
& 0,1496 \text { gr Subst. gaben } 0,1108 \mathrm{gr} \mathrm{BaSO}_{1} \\
& 0,1498 \mathrm{gr} \text { Subst. gaben } 0,0667 \mathrm{gr} \mathrm{AgCl} \\
& \mathrm{C}_{24} \mathrm{H}_{13} \mathrm{SCl} \text { Ber. C 74,85 H 4,09 } \mathrm{S} 10,00 \text { Cl 11,06\% } \\
& \text { Gef. , } 74,75 \quad, 4,49 \quad, 10,17 \quad, 11,01 \%
\end{aligned}
$$

Die Stellung des Chloratoms ergibt sich aus dem für das Benzaldehydderivat oben angenommenen Schema der Bildungsweise.

Auch in diesem Falle wurde die Menge des Kohlenoxyds quantitativ bestimmt, die bei der Darstellung des Chlorproduktes abgespalten wurde. Sie erwies sich als semimolekular, was ungefähr der erhaltenen Ausbeute an dem chlorierten Körper entspricht.

$$
\begin{aligned}
& 0,2643 \text { gr Subst. yaben } 9,6 \mathrm{gr} \text { feuchtes } \mathrm{CO}(22,50,714 \mathrm{~mm}) \\
& \mathrm{C}_{21} \mathrm{H}_{15} \mathrm{SCl}-\mathrm{CO} \text { Ber. CO } 7,63 \% \text { Gef. CO } 3,85 \% \\
& \text { Freiburg i. Lechtland, I. Chem. Lab. (L. Lniversität. }
\end{aligned}
$$

\title{
Über Stilben-0-o'-dicarbonsäure
}

\author{
von
}

Paul Ruggli und R. Ernest Meyer.

(28. XI. 21.)

Die vorliegende Arbeit behandelt eine Reihe von Derivaten der Stilben-o-o'dicarbonsäure (Formel III), deren Studium wir von dem Gesichtspunkt aus unternahmen, zu prüfen, ob man ihre $\ddot{A}$ thylendoppelbindung in eine Acetylenbindung verwandeln kann, d. h. ob sich die noch unbekannte Tolan-o-o'-dicarbonsäure (IV) aus ihr gewinnen lässt. Verbindungen mit Carboxyl in Orthostellung zur Acetylengruppe sind unseres Wissens noch nicht bekannt; ihre Darstellung würde wegen der zu erwartenden Umlagerungen wie auch in anderer präparativer Hinsicht. Interesse verdienen. Als Ausgangsmaterial für orthocarboxylierte 